\title{
Foliage Chemistry Influences Tree Choice and Landscape Use of a Gliding Marsupial Folivore
}

\author{
Kara N. Youngentob • Ian R. Wallis • \\ David B. Lindenmayer • Jeff T. Wood • \\ Matthew L. Pope • William J. Foley
}

Received: 18 May 2010/Revised: 4 November 2010 /Accepted: 18 November 2010 /Published online: 16 December 2010

(C) Springer Science+Business Media, LLC 2010

\begin{abstract}
The chemical quality of forage may determine landscape use and habitat quality for some herbivorous species. However, studies that investigate the relationship between foliar chemistry and foraging choices in wild vertebrates are rare. Petauroides volans (the greater glider) is unique among Australian marsupial folivores because it glides. It also frequently consumes foliage from both major Eucalyptus subgenera, Eucalyptus (common name "monocalypt") and Symphyomyrtus (common name "symphyomyrtle"), which differ markedly in their foliar chemistry. Such differences are thought to be a product of co-evolution that also led to guildspecific plant secondary metabolite (PSM) specialization among other marsupial eucalypt folivores. To explore whether foliar chemistry influences tree use, we analyzed foliage from eucalypt trees in which we observed $P$. volans during a radio tracking study and from eucalypt trees in which animals were never observed. We used a combination of chemical assays and near infrared spectrophotometry (NIRS) to determine concentrations of nitrogen $(\mathrm{N})$, in vitro available nitrogen (AvailN), and in vitro digestible dry matter (DDM) from foliage sampled from the monocalypt and symphyomyrtle species, and total formylated phloroglucinol compounds (FPCs) and sideroxylonals (a class of FPCs) from the symphyomyrtle species (FPCs do not occur in monocalypts). Tree size and spatially-dependent, intraspecific variations in
\end{abstract}

\footnotetext{
K. N. Youngentob $(\triangle) \cdot$ D. B. Lindenmayer $\cdot$ J. T. Wood $\cdot$

M. L. Pope

The Fenner School of Environment and Society, The Australian

National University,

Canberra ACT 0200, Australia

e-mail: kara.youngentob@anu.edu.au

I. R. Wallis • W. J. Foley

Botany and Zoology, Research School of Biology, The Australian

National University,

Canberra ACT 0200, Australia
}

sideroxylonals and DDM concentrations in the symphyomyrtle foliage and of N, AvailN, and DDM in the monocalypt species were important indicators of tree use and habitat suitability for $P$. volans. The results i) demonstrate that guild-specific PSMs do not always lead to guild-specific foraging; ii) provide a compelling co-evolutionary case for the development of gliding in P. volans; and iii) have implications for the management and conservation of this and other folivorous species.

Key Words Herbivory · Available nitrogen · Plant secondary metabolite $\cdot$ Plant-animal interaction . Eucalyptus $\cdot$ Petauroides volans $\cdot$ Specialist $\cdot$ Coevolution

\section{Introduction}

Variations in plant nutrients and herbivore-deterrent plant secondary metabolites (PSMs) across a landscape create a chemically complex environment that may influence the distribution and abundance of animal species (Braithwaite et al. 1983; Stephens and Krebs 1986; Moore et al. 2010). Identifying the factors that determine habitat suitability is essential for the conservation of species and communities. Those species that survive in a relatively narrow niche, both in terms of the range of food-types available and the variation of chemical composition that they can tolerate, may be particularly sensitive to landscape and climatic changes that can affect the quality and availability of their food (Lawler et al. 1997; Moore et al. 2004).

Eucalyptus (L'Herit) foliage contains relatively low concentrations of nutrients, particularly nitrogen $(\mathrm{N})$, but high concentrations of PSMs that can cause toxicosis and reduce the digestibility of plant nutrients (Cork and Foley 1991; Moore et al. 2004; Scrivener et al. 2004). Concentrations of 
nutrients and PSMs in eucalypts often vary considerably from tree to tree, even within the same species (Wallis et al. 2002; Andrew et al. 2005). Several studies have demonstrated how variation in $\mathrm{N}$, tannins, and formylated phloroglucinol compounds (FPCs) differentially affect feeding by Phascolarctos cinereus (the koala), Pseudocheirus peregrinus (the common ringtail possum), and Trichosurus vulpecula (the common brushtail possum) (Lawler et al. 1998a; Wallis et al. 2002; Marsh et al. 2003a, b; Moore and Foley 2005; Moore et al. 2005). It is not known whether intraspecific variations in FPCs or $\mathrm{N}$ availability influence the foraging of Petauroides volans (the greater glider, Fig. 1), a marsupial folivore that, like $P$. cinereus, feeds almost exclusively on eucalypt leaves.

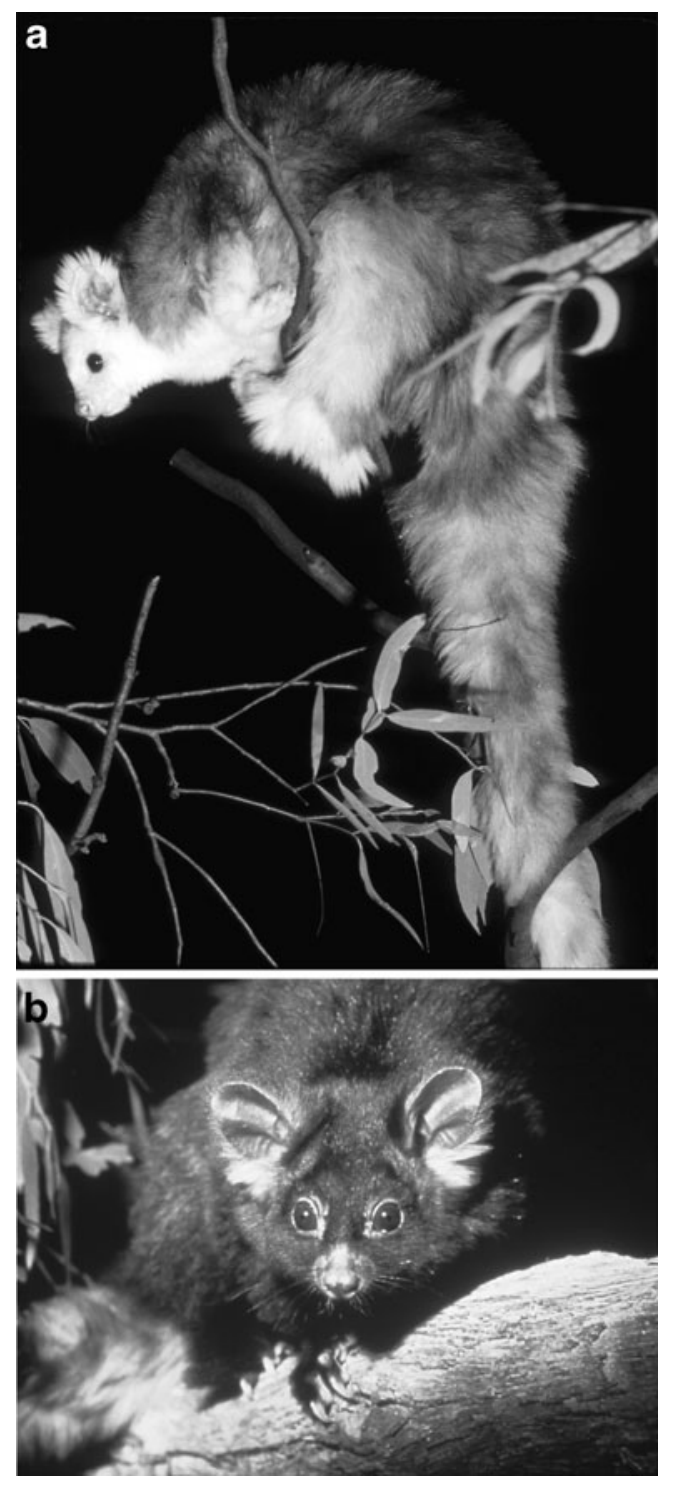

Fig. 1 The greater glider (Petauroides volans), shown here in light (a) and dark (b) color phases, is a marsupial folivore that feeds primarily on Eucalyptus leaves. Photographs by David Lindenmayer
Differential susceptibility to PSMs may reduce competition among co-occurring herbivores by restricting them to eating plants that contain the unique chemical signature they have evolved to tolerate (Moore et al. 2004). It may be appropriate to consider leaf-eating animals, not as folivores per se, but as specialists on particular metabolites. Determining the PSM tolerances of co-occurring herbivorous species also could shed light on evolutionary relationships between plants and animals and current patterns of species distributions and biodiversity in particular habitats.

In addition to intraspecific variation in foliar chemistry, there are also differences in chemistry among eucalypt species in the two major eucalypt subgenera, Eucalyptus (common name "monocalypt") and Symphyomyrtus (common name "symphyomyrtle"). Symphyomyrtle species produce FPCs that, when ingested by mammals, stimulate the serotonergic systems and probably induce sensations of nausea (Lawler et al. 1998b). When feeding on eucalypts, both $P$. cinereus and $T$. vulpecula feed mainly on symphyomyrtle species. Although tolerant of FPCs, captive animals eat less as foliar concentrations of these compounds increase (Lawler et al. 1998a; Wallis et al. 2002). Similarly, FPCs, including sideroxylonals, influence tree use by freeliving T. vulpecula and P. cinereus (Scrivener et al. 2004; Moore and Foley 2005; Moore et al. 2010). In contrast, $P$. peregrinus is relatively intolerant of FPCs and feeds primarily on monocalypt species (Lawler et al. 1998a; Moore et al. 2004).

Monocalypts do not produce FPCs (Eschler et al. 2000) but their foliage contains high concentrations of tannins (Wallis et al. 2010) that can bind to nitrogenous compounds in plant tissues and reduce the digestibility of plant proteins (Silanikove et al. 1996; Marsh et al. 2003b). Using an in vitro assay (DeGabriel et al. 2008), Wallis et al. (2010) showed that the foliage of 31 monocalypt species contained about half the available nitrogen (AvailN) of that of 82 symphyomyrtle species due to $\mathrm{N}$ binding by tannins. Tannins influence the physiology and foraging behavior of many herbivorous species, although not all marsupial folivores appear to be affected equally (Osawa and Sly 1992; Silanikove et al. 1996; Marsh et al. 2003a). When tannins are inactivated by coating leaves with polyethylene glycol (PEG), some herbivores, including T. vulpecula, eat more of the treated material, while others, such as $P$. peregrinus, do not (Marsh et al. 2003b). DeGabriel et al. (2009) showed that $\mathrm{N}$ availability in eucalypt foliage is an important determinant of reproductive success for $T$. vulpecula in the wild.

In mixed stands of eucalypt trees, representatives from both major subgenera tend to co-dominate and this is known as Pryor's Rule (Pryor 1959). Thus, one can suggest that by specializing on the PSMs produced by a particular eucalypt subgenus, folivores could inhabit the same tracts 
of forest without competing for food (Marsh et al. 2003a). In turn, coevolution between plants and animals may have influenced the diversity of plant chemotypes and foraging adaptations of herbivores (Ehrlich and Raven 1964; Thompson 1994; Futuyma 2000; Becerra 2007). As a result of such co-evolutionary relationships, guild-specific PSMs are often thought to lead to guild-specific foraging (Thompson 1994; Rausher 2001).

We focused on $P$. volans, the only Australian folivorous marsupial that glides. Although past studies have linked foliar concentrations of $\mathrm{N}$ and tannins to tree species preference and forage consumption (Foley and Hume 1987; Kavanagh and Lambert 1990; Mcllwee et al. 2001), we do not know how this animal responds to variation in the concentrations of AvailN or FPCs. Although P. volans is argued to be a monocalypt and tannin specialist, like the closely related $P$. peregrinus (Family Pseudocheiridae) (Moore et al. 2004), field studies have indicated that it regularly consumes foliage from eucalypt species of both major subgenera (Kavanagh and Lambert 1990; Cunningham et al. 2004).

We posed a series of questions to investigate the role of eucalypt foliage chemistry on tree choice, landscape use, and the habitat requirements of $P$. volans. Due to the association of particular PSMs (e.g., FPCs) with particular eucalypt subgenera, the first three questions were investigated on the monocalypt and symphyomyrtle species independently. 1) Are there significant differences in foliar concentrations of FPCs (total FPCs and sideroxylonals), N, AvailN, and digestible dry matter (DDM) among trees in which $P$. volans was and was not observed? 2) Is the relationship between $P$. volans tree use and foliar concentrations of N, AvailN, and DDM the same for trees from both major subgenera of Eucalyptus? 3) Are feedingspecific observations or spatial considerations (i.e., the distance of trees with no animal observations to trees with observations) important for determining whether there is a relationship between foliage chemistry and tree use? 4) Do our results agree with current hypotheses about the foraging niche of $P$. volans as a "specialist" folivore? 5) Do our results have implications for the conservation of $P$. volans and other folivorous species?

\section{Methods and Materials}

Study Area Our study site was $100 \mathrm{~km}$ west of Canberra in Buccleuch State Forest, New South Wales, Australia $148^{\circ}$ $40^{\prime} \mathrm{E}, 35^{\circ} 10^{\prime} \mathrm{S}$. The area includes a 50,000 ha exotic pine (Pinus radiata) plantation bordered by native eucalypt forest and grazing farmland. Extensive areas of native forest were cleared from the late 1930s through the 1980s to establish the pine plantation, leaving patches of remnant eucalypt forest (1-100 ha) embedded within the pine (Lindenmayer et al. 1999). Five neighboring eucalypt forest remnants were selected for a separate radio-tracking study to investigate $P$. volans home-range size and tree use in a fragmented forest ecosystem (Pope et al. 2004). These remnants ranged in size from 1.6 to 18.2 hectares and varied in shape, but they had similar vegetation communities dominated by narrow-leaved peppermint [Eucalyptus radiata (monocalypt)], ribbon gum [E. viminalis (symphyomyrtle)] and mountain gum (E. dalrympleana [symphyomyrtle)]. A more complete description of the study area, including a list of common canopy and understory vegetation is in Lindenmayer et al. (1999).

Radio-tracking Forty P. volans from the five neighboring eucalypt forest remnants were captured and fitted with single-stage radio-transmitter collars (Faunatech Pty Ltd, Victoria, Australia). Effort was taken to ensure that every $P$. volans in the selected remnants was included in the study (see Pope et al. 2004 for details). Tracking occurred over five consecutive nights each month from September 1997 to October 1998. Animals were tracked to one tree per night, and the tracking sequence was varied randomly (for a description of tracking methodology see Cunningham et al. 2004). Once we located a $P$. volans, we recorded its behavior (feeding, grooming, perching, mating, and not observable), tagged the tree in which it was observed, and recorded the tree location with a differentially-corrected, hand-held GPS (Global Star Pty Ltd, Melbourne).

Tree-leaf Sampling Radio-tracking data provided a unique opportunity to investigate tree use by $P$. volans in relation to recently identified PSMs and a newly described measure of forage quality (available nitrogen). A study published on tree use by wild $P$. cinereus based on tree location data recorded from 1993 to 2004 established the feasibility of sampling foliage from feeding trees identified during earlier tracking efforts (Moore et al. 2005). No large-scale changes to the selected remnant areas, such as harvesting of the surrounding pines, which might alter the concentrations of foliar chemistry, have occurred since the original radio-tracking.

In December 2006, we revisited the five fragments and relocated 261 of the original 433 trees used at night time by $P$. volans. Lost tree tags made it impossible to identify all trees from the original study. We collected leaves from all tree species in which $P$. volans was observed (see Cunningham et al. 2004 for a complete list). The vast majority of observations occurred in E. radiata (41\% of eucalypt tree use) and E. viminalis-E. dalrympleana (35\%) (Cunningham et al. 2004; Pope et al. 2004). Due to the relatively small number of observations in other species, we focused our analysis solely on these three tree species. Data for $E$. 
viminalis and $E$. dalrympleana were pooled during the radio-tracking study due to the difficulty of distinguishing these closely related trees in the field. Both species produce FPCs, and both are eaten by $P$. volans, so grouping them also was appropriate for this study.

We collected foliage from one or more untagged neighboring trees of the same species and similar size $[ \pm 10 \%$ diam. at breast height (DBH) $]$ as each numbered tree $(N=112)$. Cunningham and others (2004) found a correlation between tree size and tree use by $P$. volans, so we selected similar-sized trees to minimize the effect of this potentially confounding factor. If a tree did not have a suitable neighbor within $20 \mathrm{~m}$, we did not sample a neighbor. Where possible, we also sampled canopy trees from the few areas within the remnant patches where no $P$. volans were observed. These trees were more than $20 \mathrm{~m}$ from any other tree with an animal observation $(N=53)$. The purpose of this sampling strategy was to document the chemical composition of eucalypts within the proximity of the animals' home-ranges without introducing a potentially confounding distance factor. The aim of the original radiotracking study was to determine home-range size rather than total tree use (Pope et al. 2004). Animals invariably used trees in addition to those in which they were observed. We recognize that $P$. volans may have used some of the "no observations" trees, though we assumed a bias towards a non-use status for trees in which we did not observe an animal.

We obtained leaf samples by shooting a branch from the top half of the canopy using a 0.222 caliber rifle, and then recorded tree height and DBH at $1.3 \mathrm{~m}$ from the forest floor on the uphill side of the tree by using a laser clinometer and a measuring tape, respectively. We collected about $50 \mathrm{~g}$ of fully expanded, healthy adult leaves from each tree. Petauroides volans feeds on both young and mature foliage but we chose mature foliage because concentrations of both $\mathrm{N}$ and PSMs can change considerably as a leaf ages (Choo et al. 1981; Kavanagh and Lambert 1990). Thus, selecting mature foliage - that available for most of the yearensured that leaf phenology did not obscure the relative concentrations of compounds between trees. We placed the leaf samples in a portable freezer (Engel MT17: Brisbane, Australia) and transported them to the laboratory where they were freeze-dried and then ground to pass a $1 \mathrm{~mm}$ sieve using a Cyclotec 1093 mill (Tecator, Sweden).

NIR Spectrometry We placed samples of freeze-dried, ground leaf in an oven $\left(40^{\circ} \mathrm{C}\right)$ for $12 \mathrm{~h}$ to equilibrate atmospheric moisture. After cooling in a desiccator, we recorded the spectra $(400 \mathrm{~nm}$ to $2,500 \mathrm{~nm}$ at $2-\mathrm{nm}$ intervals) with a NIRSystems Model 6500 Scanning Spectrophotometer (Foss NIRSystems, Laurel, MD, USA) fitted with a spinning cup module. The spectrophotometer was housed in a room at $22^{\circ} \mathrm{C}-24^{\circ} \mathrm{C}$ and at $55 \%-60 \% \mathrm{RH}$. We checked the accuracy of measurements at regular intervals by using a standard of known chemical composition. We converted reflectance spectra $(\mathrm{R})$ to absorbance values $[A=\log (1 / R)]$ and transformed them to a firstderivative spectrum to emphasize changes in slope and reduce variation resulting from differences in particle size (Osborne et al. 1993). We collected spectra from each sample in duplicate, and these were averaged once the spectral noise [the root mean square of the difference between replicates $\left.(1 / \mathrm{R}) / 10^{6}\right)$ ] was less than 250 .

We determined foliar concentrations of $\mathrm{N}$, available $\mathrm{N}$, and DDM on a calibration set of 100 samples selected to represent the population of all sampled eucalypts based on a Mahalanobis distance calculation of spectral variation (WinISI II V. 1.02a; InfraSoft International, Port Matilda, PA, USA). We developed calibration equations by using a modified partial least squares regression (MPLS: Shenk and Westerhaus 1991), and a standard normal variate and detrend scatter correction (SNV detrend). We tested various combinations of Savitzy-Golay derivative-based spectral smoothing functions provided by the ISI software (Win ISI; Port Matilda, PA, USA) to remove unnecessary spectral signal components and to improve the accuracy of the prediction models. We report the best results from the application of these mathematical transformations in Table 1. The use of MPLS requires cross-validation to prevent over-fitting the model for which we used a leave-oneout (LOO) resubstitution method. The LOO cross-validation

Table 1 Results from MPLS regression with cross-validation for modeling the relationship between spectral characteristics of eucalypt foliage and foliar concentrations of nitrogen $(\mathrm{N})$, available nitrogen (AvailN), and digestible dry matter (DDM)

\begin{tabular}{lcccccrrr}
\hline Constituent & SNV detrend + math treatment & $N$ & Mean & SD & SEC & $R^{2}$ & SECV & $1-V R$ \\
\hline $\mathrm{N}$ & 2841 & 96 & 1.21 & 0.20 & 0.04 & 0.97 & 0.06 \\
AvailN & 1441 & 78 & 0.37 & 0.31 & 0.06 & 0.96 & 0.10 \\
DDM & 1441 & 89 & 0.44 & 0.12 & 0.02 & 0.97 & 0.03 & 0.93 \\
\hline
\end{tabular}

Math treatment refers to the Savitzy-Golay derivative based spectral smoothing and includes the derivatives and the number of data points across which the smoothing functions were calculated; SEC is standard error of calibration and SECV is standard error of cross-validation predictions; the degree of correlation between predicted and actual measured values is indicated by the $R^{2}$ and by 1 -VR for the cross-validation data. The prediction equation and validation are based on all species of Eucalyptus collected. 
provides an estimate of model error based on data resampling (Elisseeff and Pontil 2002). We divided data into $\mathrm{n}$ groups and left one group out of the model in rotation so that the learning algorithm was trained multiple times on the n-1 group, while the remaining group tested the model's accuracy. We generated a standard error of cross-validation by pooling residuals from each prediction (Table 1). Finally, we validated the approach for $\mathrm{N}$ using 20 additional tree-leaf samples not included in the prediction model.

Chemical Assays We used freeze-dried ground material for all analyses and corrected for residual moisture by drying $1.00 \mathrm{~g}$ of material to constant mass at $50^{\circ} \mathrm{C}$. The $\mathrm{N}$ content of foliage was determined on duplicate samples (250土 $10 \mathrm{mg}$ ) using a semi-micro Kjeldahl technique calibrated with ammonium sulphate. We determined in vitro DDM and AvailN using the method of DeGabriel et al. (2008). This involved digesting the samples in porous bags (Ankom F57, Ankom Technology, Macedon, NY, USA), first with pepsin $(24 \mathrm{~h})$ and then with cellulase (48 h) to mimic digestion in a hindgut-fermenting herbivore. The purpose of the assay was to rank trees with regard to AvailN and DDM rather than trying to give precise in vivo values for these constituents. We discarded values if the coefficient of variation between duplicates was more than $2 \%(\mathrm{~N})$ or $7 \%$ (AvailN and DDM) and repeated the assay.

We extracted formylated phloroglucinol compounds by sonicating single $50 \mathrm{mg}$ foliage samples with a known mass (ca $4.0 \mathrm{~g}$ ) of solvent ( $7 \%$ water in acetonitrile containing $0.1 \%$ trifluoroacetic acid and $0.3000 \mathrm{~g}$ per liter of the internal standard 2-ethylphenol) for 5 min (Wallis and Foley 2005). The resulting mixture was filtered (0.2 um) directly into an autosampler vial and then we injected $20 \mathrm{ul}$ onto a Wakosil $250 \times 4 \mathrm{~mm}$ GL 3C18RS (SGE Analytical: Ringwood, Australia) column maintained at $37^{\circ} \mathrm{C}$ with a flow rate of $0.75 \mathrm{ml} / \mathrm{min}$ on a Waters Alliance Model HPLC. We eluted the FPCs under gradient conditions with $0.1 \%$ TFA acid in water (A) and $0.1 \%$ TFA in acetonitrile (B) as follows: $40 \% \mathrm{~A} / 60 \% \mathrm{~B}$ for 5 min, linear gradient to $90 \% \mathrm{~B} / 10 \% \mathrm{~A}$ at $60 \mathrm{~min}$, held for $10 \mathrm{~min}$ and returned to starting conditions over $10 \mathrm{~min}$. We measured the peak response at $275 \mathrm{~nm}$. We quantified the specific FPCs present by using authentic standards purified in the laboratory. The coefficient of variation between duplicate measurements is typically less than $4 \%$ (Wallis et al. 2010).

Statistical Analyses We conducted statistical analyses separately on the Eucalyptus (monocalypt) and Symphyomyrtus (symphyomyrtle) subgenera. We compared foliar concentrations of N, AvailN, and DDM among the different tree categories. These were: 1) trees in which we observed $P$. volans feeding once (feeding observation); 2) trees in which we saw an animal feeding multiple times (multiple feeding observations); 3) trees in which we observed $P$. volans in a non-feeding activity only (non-feeding observation); 4) neighboring trees with no animal observations (no observation neighbor); and 5) trees more than $20 \mathrm{~m}$ from any tree in which we observed an animal (no observation area). We also compared foliar concentrations of total FPCs and sideroxylonals (a class of FPC) among these tree-use categories for the symphyomyrtle species.

We combined feeding and multiple feeding trees for the following analyses due to the relatively small number of trees in each category. We assessed the distribution of data with a normal-quantile plot. Where data were normally distributed, we used ANOVAs to test the hypothesis that two population means were equal. We used a KruskalWallis test when the normality assumption for ANOVAs could not be met and when our samples sizes were less than 30 but greater than 14 (Hollander and Wolfe 1999). Larger trees were less likely than smaller ones to have a suitable neighbor within a $20 \mathrm{~m}$ radius. Therefore, despite our effort, this resulted in a disproportionate sampling of smaller trees in the no observation categories when compared to some of the observation categories. Where significant differences were observed for several potentially confounding variables, we used logistic, binomial Generalized Linear Models (GLMs) to investigate whether a significant difference in a chemical constituent concentration remained once we controlled for other significant explanatory variables, such as the DBH.

Monocalypt trees in which we saw $P$. volans feeding repeatedly did not appear to have the same variance for $\mathrm{N}$ as monocalypt trees with no observations. There were sufficient multiple feeding observation trees in this monocalypt group to investigate the trend by using nonparametric statistics. First, we used an Ansari-Bradley test to determine equality of variance (Hollander and Wolfe 1999). To test whether animals avoided feeding repeatedly on trees with low concentrations of $\mathrm{N}$, we determined the number of trees with no $P$. volans observations that had a lower concentration of $\mathrm{N}$ than any tree with multiple feeding observations. We then calculated the probability of this number or a greater number occurring by chance at either extreme of the distribution.

\section{Results}

Foliar Chemistry and Measured Tree Attributes We successfully modeled the relationship between absorbance spectra from eucalypt foliage and foliar concentrations of $\mathrm{N}$, AvailN, and DDM (Table 1). The independent validation of $\mathrm{N}$ samples $(N=20)$ resulted in excellent agreement between the predicted and analyzed values $\left(R^{2}=0.96\right.$; 
$R M S E=0.042$ ). This suggested that the samples we selected for the training algorithm by the Mahalanobis distance calculation method were representative of the spectral range of our data.

We illustrate the distributions of data for each variable according to eucalypt subgenera, monocalypt and symphyomyrtle (Fig. 2). These distributions were further segregated by tree use category (no observation area, no observation neighbor, non-feeding observation, feeding observation, and multiple feeding observations). The symphyomyrtle species (E. viminalis and $E$. dalrympleana) had higher concentrations of AvailN $(P<.001, t=22.94$, mean of the differences $=$ $0.48 \%$ dry matter $(\mathrm{DM}))$ and DDM $(P<.001, t=27.96$, mean of the differences $\left.=0.19 \mathrm{~g} . \mathrm{g}^{-1} \mathrm{DM}\right)$ than did the monocalypt
Monocalypt
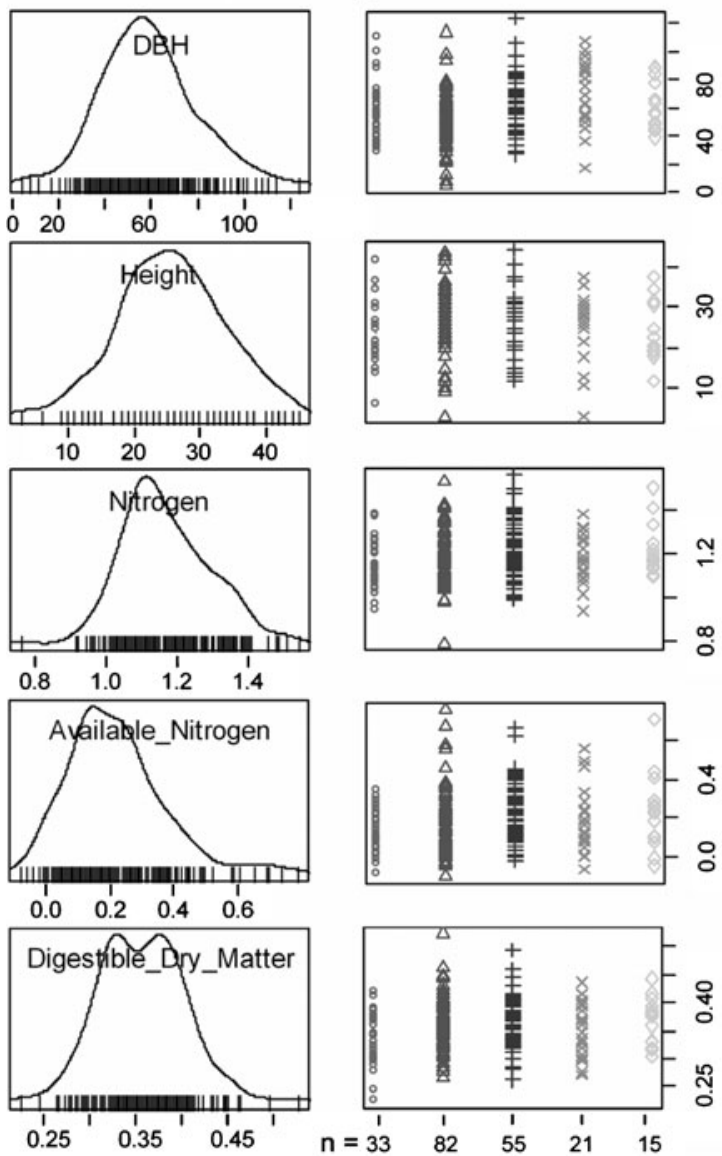

No observation area

No observation neighbour

Non-feeding observation

Feeding observation

Multiple feeding observations

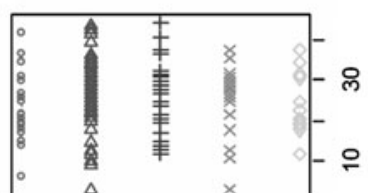

\section{$\infty$}
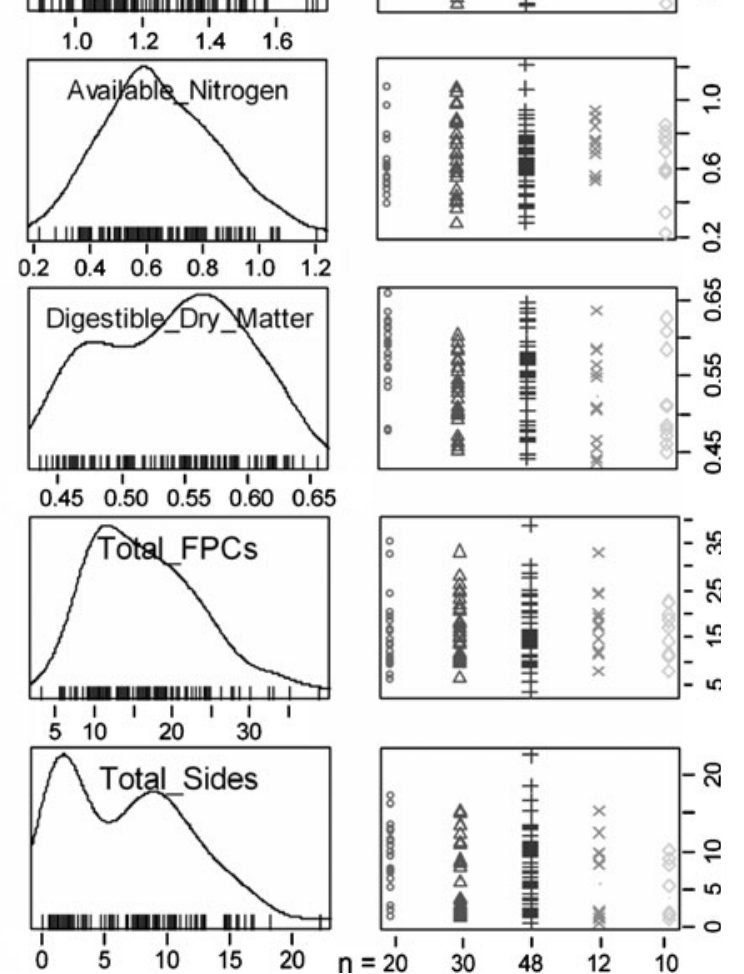

(DM)), available nitrogen (AvailN; \% DM), digestible dry matter (DDM; g. $\mathrm{g}^{-1} \mathrm{DM}$ ), total formylated phloroglucinol compounds (total FPCs; mg. ${ }^{-1}$ DM), and sideroxylonals (Total Sides mg.g ${ }^{-1} \mathrm{DM}$ ). The sample size for each tree category is provided under the tree category distributions 
species ( $E$. radiata) ( $P$-values from Student's $t$-test of means not included in Fig. 2).

We explored the interactions among the data by using a Pearson's correlation matrix (Table 2). The correlation between the concentrations of $\mathrm{N}$ and AvailN in the monocalypts was much lower $(r=0.462, P<0.001)$ than in the symphyomyrtles $(r=0.914, P<0.001)$. The monocalypts also showed a weak positive correlation between $\mathrm{N}$ and DDM $(r=0.170, P=0.02)$. This relationship was negative in the symphyomyrtles $(r=-0.602, P<0.001)$. Likewise, the concentration of AvailN was positively correlated with DDM in the monocalypt species $(r=$ $0.617, P<0.001)$ and negatively correlated in the symphyomyrtles $(r=-0.445, P<0.001)$. When we converted tree use category to an ordinal variable, which assumed a linear relationship of increasing preference as a feeding tree from 0 to 4 (Table 2), we identified a significant correlation between tree use category and $\mathrm{DBH}$ for both the monocalypt $(r=0.206, P=0.003)$ and symphyomyrtle $(r=0.376, P<0.001)$ classes and between tree use category and tree height for symphyomyrtles $(r=0.321, P<0.001)$. We also observed a weak but significant correlation between tree use category and AvailN $(r=0.146, P=0.03)$ for the monocalypts and between tree use category and sideroxylonals $(r=-0.242, P=0.008)$ in the symphyomyrtles. We identified a negative correlation for both $\mathrm{N}$ and AvailN with sideroxylonals $(r=0.575, P<0.001$ and $r=$ $0.519, P<0.001$, respectively) and a positive correlation for $\mathrm{N}$ and AvailN with total FPCs $(r=0.669, P<0.001$ and $r=0.713, P<0.001$, respectively).
Tree Use by P. volans When comparing all symphyomyrtle trees in which we observed $P$. volans to trees with no observations, only DBH $(P<0.001)$ and height $(P<0.001)$ differed significantly (Table 3 ). Monocalypt trees in which we saw animals also had a larger DBH $(P<0.001)$ than did those in which we did not observe $P$. volans. A GLM that included both DBH and AvailN (near significant in monocalypt means test, $P=0.058$ ) suggested that both variables were important in explaining the difference among monocalypt trees in which an animal was, and was not, observed $(P<0.001$ and $P=0.042$, respectively) (Table 4).

We separated the trees in which we did not observe $P$. volans into two categories: "no observation neighbor" and "no observation area". Trees from areas where we did not observe $P$. volans explained most of the chemical differences among categories (Table 3). Size was the only significant difference among neighboring trees in which an animal was not observed and trees in which an animal was observed. Despite our attempt to control for these differences through our sampling criteria, symphyomyrtle trees with an animal observation were significantly taller $(P<$ $0.001)$ and had a larger DBH $(P<0.001)$ than did neighboring trees in which we did not see an animal. Monocalypt neighbors also had a smaller DBH $(P<0.001)$ compared to the adjacent monocalypts in which we observed P. volans.

We found a similar difference in average height and DBH among trees sampled from areas of the forest patches where we did not observe any $P$. volans and trees in which we saw an animal (Table 3 ). In addition, both monocalypt

Table 2 Pearson's correlation coefficients among tree attributes

\begin{tabular}{|c|c|c|c|c|c|c|c|}
\hline Constituent & Subgenus & AvailN & $N$ & DDM & $\mathrm{DBH}$ & Height & Tree use category \\
\hline \multirow[t]{2}{*}{$\mathrm{N}$} & Sym & 0.914 & & & & & \\
\hline & Mon & 0.462 & & & & & \\
\hline \multirow[t]{2}{*}{ DDM } & Sym & -0.445 & -0.602 & & & & \\
\hline & Mon & 0.617 & 0.170 & & & & \\
\hline \multirow[t]{2}{*}{ DBH } & Sym & -0.024 & 0.009 & -0.138 & & & \\
\hline & Mon & -0.019 & 0.063 & -0.020 & & & \\
\hline \multirow[t]{2}{*}{ Height } & Sym & 0.068 & 0.054 & -0.131 & 0.570 & & \\
\hline & Mon & 0.040 & 0.051 & -0.041 & 0.348 & & \\
\hline \multirow[t]{2}{*}{ Tree use category } & Sym & 0.027 & 0.072 & -0.265 & 0.376 & 0.321 & \\
\hline & Mon & 0.146 & 0.055 & 0.113 & 0.206 & 0.028 & \\
\hline Total FPCs & Sym & 0.713 & 0.669 & -0.532 & -0.008 & 0.124 & 0.009 \\
\hline Sideroxylonals & Sym & -0.519 & -0.575 & 0.667 & -0.073 & -0.044 & -0.242 \\
\hline
\end{tabular}

Correlations among measure tree attributes for sampled eucalypt subgenera Eucalyptus (Mon) and Symphyomyrtus $($ Sym). Significance $(P<0.05)$ is indicated in bold. Tree use category ranked from $0-4(0=$ no observation area, $1=$ no observation neighbor, $2=$ non-feeding observation, $3=$ feeding observation, $4=$ multiple feeding observations). Measured variables include diam at breast height $(\mathrm{DBH}$; $\mathrm{cm})$, height $(\mathrm{m})$, and foliar concentrations of nitrogen (N; \% dry matter (DM)), available nitrogen (AvailN; \% DM), digestible dry matter (DDM; g.g ${ }^{-1}$ DM), total formylated phloroglucinol compounds (total FPCs; mg.g ${ }^{-1} \mathrm{DM}$ ), and sideroxylonals (mg. $\mathrm{g}^{-1} \mathrm{DM}$ ). 


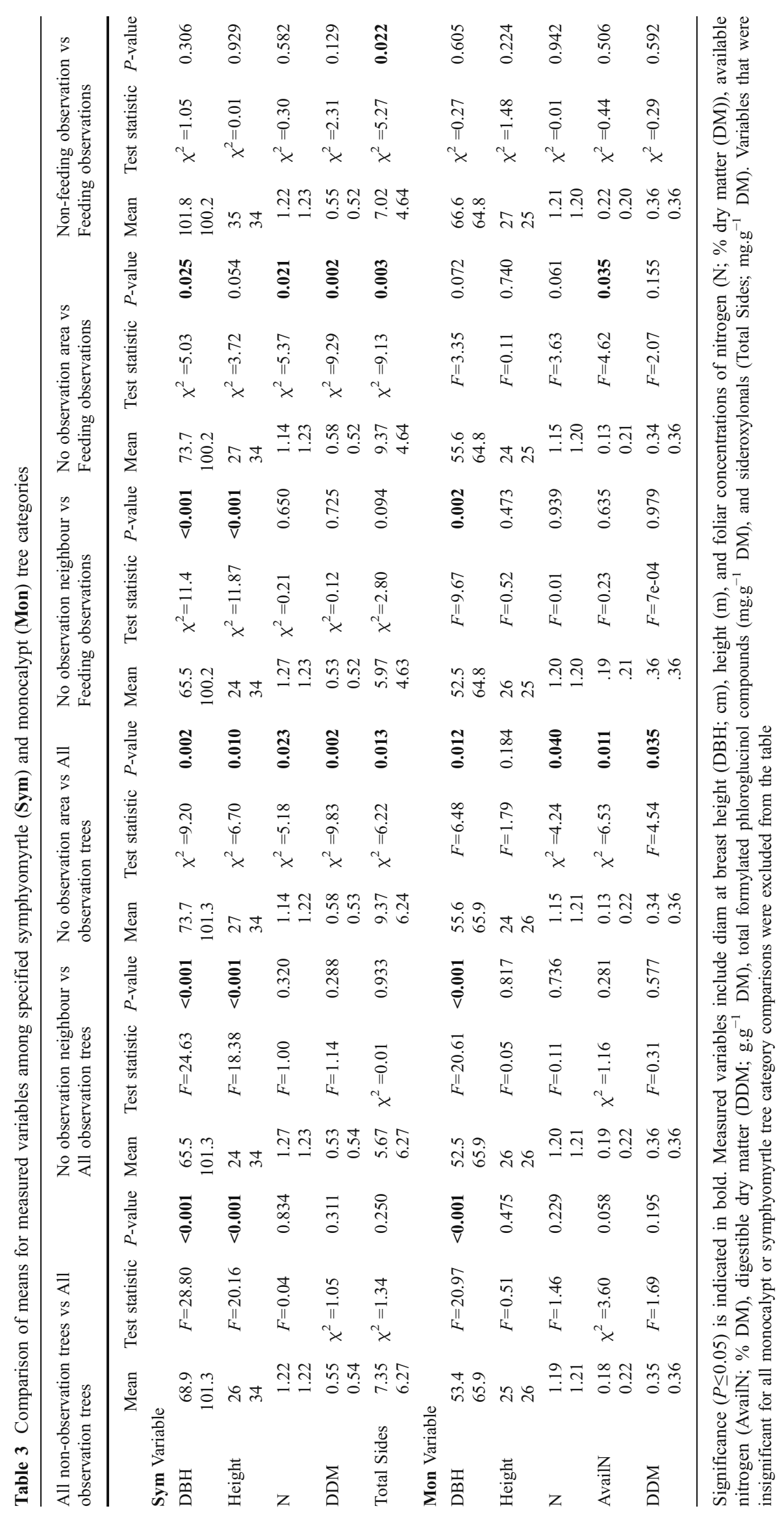




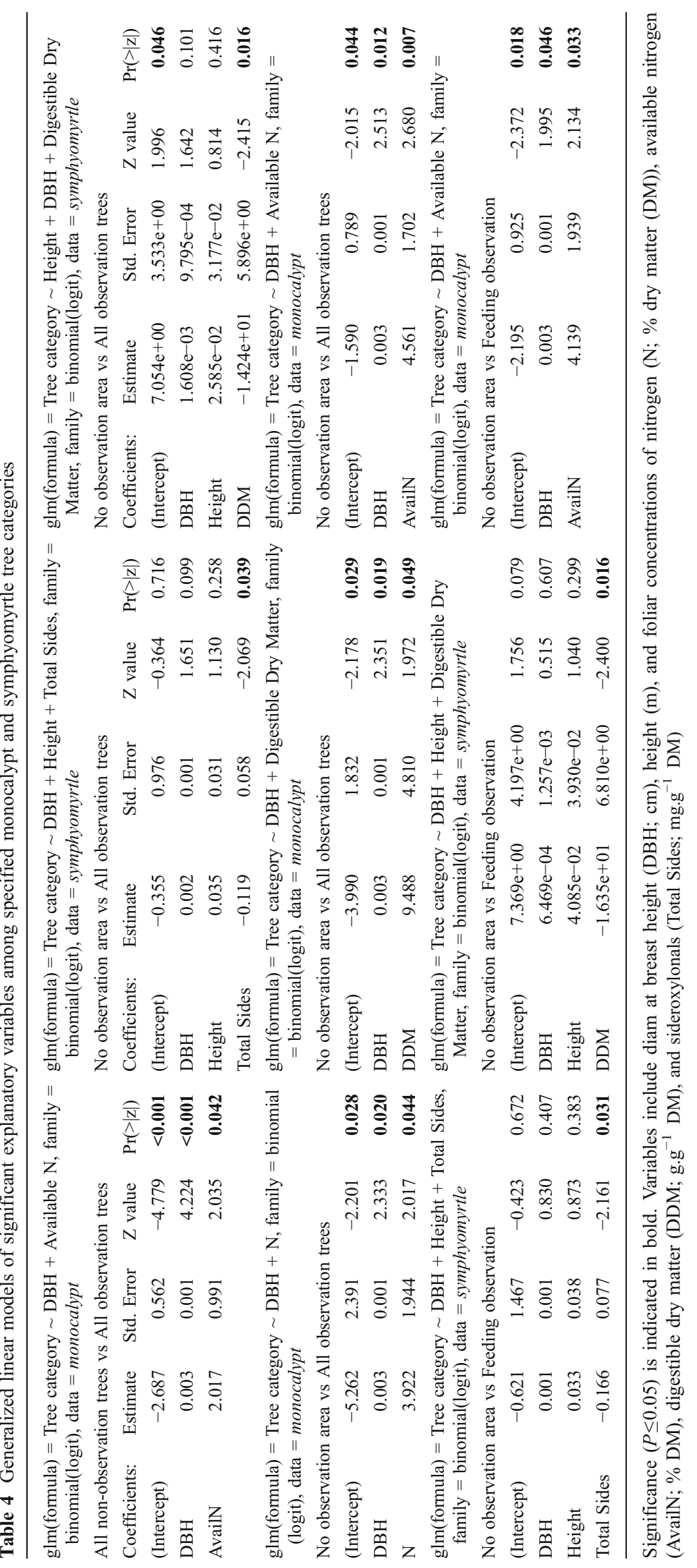


and symphyomyrtle trees from these two categories differed chemically. The GLMs that controlled for potentially confounding size variables showed that sideroxylonals $(P=0.039)$ and DDM $(P=0.016)$ explained a significant amount of the variation among symphyomyrtle tree populations from areas where $P$. volans was and was not observed (Table 4). Likewise, N $(P=0.044)$, available N $(P=0.007)$, and DDM $(P=0.049)$ explained differences in tree use between these two tree categories for monocalypt trees.

Symphyomyrtle trees in which we observed $P$. volans feeding were taller $(P<0.001)$ and had a larger DBH $(P<$ $0.001)$ than neighboring trees with no animal observations (Table 3). Monocalypt trees from the same categories differed only in mean DBH $(P=0.002)$. We found this same trend in height $(P=0.054)$ and DBH $(P=0.025)$ among symphyomyrtle trees sampled from areas where we did not observe $P$. volans, and among trees in which we observed $P$. volans feeding. Monocalypt trees from these categories had no significant difference in DBH $(P=0.072)$. Allowing for tree size, GLMs showed that symphyomyrtle trees with feeding observations had lower concentrations of sideroxylonals $(P=0.031)$ and DDM $(P=0.016)$ than did trees from areas where $P$. volans was not observed (Table 4). Similarly, GLMs showed that monocalypt trees in which we observed $P$. volans feeding were likely to have more AvailN $(P=0.033)$ and have a larger DBH $(P=0.046)$ than trees sampled from areas where we did not see any animals. A monocalypt GLM that included $\mathrm{N}$ and DBH showed no significant values for either attribute ( $P=0.096$ and $P=0.071$, respectively).

Symphyomyrtle trees in which we observed an animal feeding had lower concentrations of sideroxylonals $(P=$ 0.022 ) than did trees in which we observed $P$. volans in a non-feeding activity only (Table 3). Monocalypt trees from these categories did not differ chemically.

Monocalypt trees in which we repeatedly observed $P$. volans feeding had less variable $\mathrm{N}$ concentrations $(P=$ 0.041) than did trees in which we did not observe the animal. It appears that $P$. volans avoided feeding repeatedly from trees with $\mathrm{N}$ concentrations that reflected the lower range of measured $\mathrm{N}$ values - as found in the distribution of foliar $\mathrm{N}$ among all no observation trees $(P=0.048)$ and no observation area trees $(P=0.017)$.

\section{Discussion}

Tree Use and Foliar Chemistry The eucalypt trees in which we observed $P$. volans differed chemically from trees of the same species in nearby areas with no animal observations. In addition, symphyomyrtle trees in which animals fed differed chemically from the symphyomyrtle trees in which we observed $P$. volans engaged in a non-feeding activity only. Our findings refute the null hypothesis that there are no chemical differences among trees in which we observed $P$. volans and those in which we did not. The study demonstrates that intraspecific variations in sideroxylonals and DDM concentrations in the foliage of $E$. viminalis and E. dalrympleana and of N, AvailN, and DDM in E. radiata, play a role in tree use and hence habitat suitability for $P$. volans.

Subgenera Dependent Responses of P. volans to Foliar Chemistry The foliage of trees from the two major eucalypt subgenera pose different problems for foraging animals. The lower average concentrations of AvailN in the monocalypt, $E$. radiata, compared with the symphyomyrtles, $E$. viminalis and $E$. dalrympleana, may explain why this constituent had a significant positive relationship with $P$. volans tree use in the monocalypt species that was not seen in the other subgenus. Our data also suggest that $P$. volans may not forage repeatedly in E. radiata trees with concentration of foliar $\mathrm{N}$ below a particular threshold (around 1\% dry matter). This finding is consistent with other research indicating that foliar $\mathrm{N}$ may be a limiting environmental factor for P. volans (Foley and Hume 1987; Kavanagh and Lambert 1990). In contrast, the concentration of sideroxylonals was more important than was any measure of $\mathrm{N}$ for influencing the use of $E$. viminalis and $E$. dalrympleana.

The concentration of DDM played opposing roles in explaining differences among tree categories for monocalypts and symphyomyrtles. This measure of forage digestibility is governed largely by a negative relation to concentrations of foliar lignin and phenols (e.g., tannins) (Choo et al. 1981). Presumably, the indigestible dry matter of monocalypts contains much of the foliar $\mathrm{N}$ bound by tannins and as a result, DDM had a stronger positive correlation to foliar concentrations of AvailN than N. This supports the pattern of tree use by $P$. volans when foraging in E. radiata; they should seek trees whose foliage contains lower concentrations of tannins and thus higher concentrations of available nitrogen.

In contrast, we observed a negative relationship between DDM and concentrations of both $\mathrm{N}$ and AvailN in the symphyomyrtle species. We also found a positive correlation between the concentrations of DDM and sideroxylonals. These correlations create a nutritional dilemma for animals eating those leaves because the most digestible components of the foliage contain the most sideroxylonal but the least $\mathrm{N}$ and AvailN, which may be bound to other non-digestible lignin compounds (Cork and Foley 1991; Dyckmans et al. 2002). We would not expect this strong negative relationship between DDM and $\mathrm{N}$ in immature leaves, which have a higher ratio of $\mathrm{N}$ to ligno-cellulose than do mature leaves (Kavanagh and Lambert 1990; Cork 
and Foley 1991). The age variability of this ratio is greater for $E$. viminalis than for other species like E. radiata and, not surprisingly, some field studies have reported that $P$. volans will forage preferentially on young leaves, particularly from E. viminalis (Kavanagh and Lambert 1990). We propose that the opposing roles that DDM played in explaining $P$. volans tree use in the two subgenera results from the relationship between DDM and these other foliage constituents.

Relevance of Feeding Observations and Spatial Considerations for Determining Relationship between Foliar Chemistry and Tree Use Specific observations of feeding strengthened the negative relationship between the choice of feeding tree and the sideroxylonal concentration of foliage for E. viminalis and E. dalrympleana. Feedingspecific observations also were useful in identifying significant differences in the variance of $\mathrm{N}$ concentrations among tree categories for the monocalypt species. In most instances, however, feeding observations were not required to identify chemical differences among tree categories. This concurs with Moore and Foley (2005), who showed that we could learn much about the chemistry of trees that were preferred by $P$. cinereus from animal location data alone.

We found that the proximity of sampled trees to any tree in which P. volans was observed was important to identify a relationship between foliar chemistry and tree use. Most of the differences among trees in which we saw an animal and those in which we did not were apparent only when the "no observation trees" were more than $20 \mathrm{~m}$ from any tree with an observation. This suggests a spatial genetic effect similar to that shown for the concentration of PSMs in the foliage of E. melliodora, whereby individual trees within $20 \mathrm{~m}$ of each other tended to be more closely related and thus more similar chemically than more distant trees (Andrew et al. 2007).

Differences in the physical characteristics of the monocalypt and symphyomyrtle species that we examined may affect spatial distributions and influence their use by $P$. volans. Both $E$. viminalis and E. dalrympleana grow taller and wider leaving more space between mature trees than typically occurs for E. radiata. Consequently, there were fewer appropriate neighbor trees for the symphyomyrtles than for the monocalypts. In addition, symphyomyrtle trees in which we saw $P$. volans in activities other than feeding often were more than $20 \mathrm{~m}$ from any feeding-observation tree. These differing spatial relationships could explain why non-feeding and feeding tree categories differed only for the symphyomyrtles.

Foraging Niche of $P$. volans Herbivores that forage primarily from a single plant genus or family, such as $P$. volans, are often labeled as "specialists" (Lindenmayer
1997; Shipley et al. 2009). However, chemical and physical attributes that can vary substantially among plants within the same species and that may occur across plant taxa generally show a stronger association to herbivore dietary specialization than does plant taxonomy (Shipley et al. 2009). In hypothesizing about the foraging niche of marsupial eucalypt folivores, Moore et al. (2004) placed P. volans with $P$. peregrinus, a monocalypt and tannin specialist that also consumes non-eucalypt foliage. Pseudocheirus peregrinus is the smallest marsupial folivore and uses caecotrophy and a specialized caecum to free $\mathrm{N}$ from tannins in the gut (McArthur and Sanson 1991; Marsh et al. 2003a). Consequently, laboratory studies have found only a weak relationship between feeding choices and concentrations of tannins for this species (Marsh et al. 2003b). Petauroides volans also feeds on tannin-rich monocalypt species, and both species can survive on a diet of monocalypt foliage in captivity (Chilcott and Hume 1984; Foley and Hume 1987). We found, however, that the activity of tannins, as indicated by the concentration of AvailN in the leaves, strongly influenced its tree use. This suggests that $P$. volans is more sensitive to tannins and their influence on $\mathrm{N}$ availability than is $P$. peregrinus. If $P$. volans is not as efficient at liberating tannin bound $\mathrm{N}$, then it may need to feed on the more AvailN-rich symphyomyrtle foliage, particularly when $\mathrm{N}$ requirements increase during pregnancy or lactation or in sites where low soil $\mathrm{N}$ is reflected in low foliar $\mathrm{N}$. It is important to recognize that this hypothesis is based on the comparison of an in vivo laboratory study with a field study that relied on in vitro methodology and requires further laboratory investigations to verify.

Petauroides volans, like other eucalypt folivores, can consume foliage containing sideroxylonals, although it showed a strong preference for foliage with lower concentrations of these chemicals. In addition, both E. viminalis and E. dalrympleana contain low concentrations of sideroxylonals, compared with some symphyomyrtle species that other arboreal folivores eat that contain high levels of sideroxylonals but no other FPCs (e.g., E. melliodora and E. polyanthemos; Wallis et al. 2002). We would not expect $P$. cinereus or $T$. vulpecula to feed selectively when faced with the concentrations of sideroxylonals that influenced tree choice in P. volans (Lawler et al. 1998a; Wallis et al. 2002). Unlike $P$. cinereus, $P$. volans also showed a stronger response to the concentration of sideroxylonals than to total FPCs (Moore et al. 2005). Laboratory feeding studies are necessary to determine whether $P$. volans is less sensitive to other FPC derivatives, such as macrocarpals. In addition, research is necessary to determine how concentrations of PSMs and nutrients influence feeding behavior on immature leaves, since we conducted our analyses on mature leaf samples only. We do not know whether $P$. volans, like $P$. cinereus and $T$. vulpecula, can persist on a diet of 
symphyomyrtle foliage alone (Moore et al. 2004); however, it appears that $P$. volans is very susceptible to the physiological effects of sideroxylonals while also lacking the adaptations of $P$. peregrinus to cope with high levels of tannins.

Studies of captive animals may show that the strategy of $P$. volans is intermediate between that of $P$. peregrinus, which specializes on foliage containing large concentrations of tannins, and that of $P$. cinereus, which specializes on foliage with significant concentrations of FPCs. In terms of PSM specialization, we propose that $P$. volans is more of a generalist than $P$. peregrinus or $P$. cinereus. A test of the detoxification limitation hypothesis (reviewed by Marsh et al. 2006) should reveal that $P$. peregrinus and $P$. cinereus have a higher rate of foliage consumption and detoxification of PSMs from their preferred subgenus than does $P$. volans. However, $P$. volans should be able to eat more when presented with foliage from preferred monocalypt and symphyomyrtle species simultaneously, each requiring different PSM detoxification pathways. In other words, $P$. volans will eat foliage from the symphyomyrtle species until the physiological effects of the FPCs force it to stop. Because monocalypts do not contain FPCs, $P$. volans can consume the foliage of $E$. radiata while detoxification processes remove the FPCs. It then can revert to eating symphyomyrtle foliage before ingesting debilitating amounts of tannins in the monocalypts (Marsh et al. 2006).

Petauroides volans may use this strategy to maintain its nutrient and energy requirements despite its small body size and poor-quality diet. Observations of free-living P. volans in our study area suggest that it may divide its feeding time between monocalypt and symphyomyrtle tree species (Cunningham et al. 2004). Energy and detoxification requirements, coupled with the general paucity of nutrients in eucalypt foliage, may have favored the development of a more energy efficient means of moving through the forest, namely gliding. This adaptation also would enable $P$. volans to take advantage of favorable variations in foliage chemistry as a result of leaf age and/or distribution of genetic variability in both major eucalypt subgenera at a minimal energy cost compared to other forms of movement (Jackson 2000).

Conservation Implications Factors that confine an animal's use of the landscape, such as a highly selective diet, make them particularly susceptible to landscape change. Petauroides volans and other herbivores that persist on nutritionally-poor foliage or foliage that contains high levels of certain PSMs may be vulnerable to environmental changes that could further reduce the nutritional quality or increase the toxicity of their forage (Lawler et al. 1997; Zvereva and Kozlov 2006). Higher levels of atmospheric $\mathrm{CO}_{2}$ have been shown to increase the production of tannins and reduce the concentration of $\mathrm{N}$ in plant tissues (Lawler et al. 1997; Gleadow et al. 1998; Mansfield et al. 1999). Thus, some plants grown under elevated $\mathrm{CO}_{2}$ have reduced herbivore damage (Knepp et al. 2005). The potential effects of climate change on nutrient availability and plant toxicity need study (Lindroth 2010; Pinto et al. 2010).

We recommend that land managers give preference to conserving tracts of eucalypt forest where marsupial folivores occur. Areas where animals are not sighted may not have the same capacity to support them for several reasons, including inadequate forage quality. The ability to accurately measure variation in plant nutrients and PSMs on a landscape scale could become an important conservation tool. Large-scale mapping of foliage chemistry could help to identify areas that are suitable or unsuitable habitat for certain animals and to monitor changes to the chemical quality of landscapes that could influence their ability to support some species.

Acknowledgements The authors thank Dr. Karen Marsh for assistance with the field and laboratory components of this research. We also thank Nicole Coggan, Sarah Ugalde, Jeff Whiting, and Stewart Archer for help in the field. Two reviewers provided constructive comments that improved our original manuscript. This research was made possible by the support of The Hermon Slade Foundation, The Wilderness Society, Ecological Society of Australia, The Fenner School of Environment and Society and Botany and Zoology in the Research School of Biology at Australian National University. This research was conducted with the permission of State Forests New South Wales (permit CO32438) and National Parks (permit S12036). This project was approved by the Animal Experimentation Ethics Committee at Australian National University (project number C.RE.47.06).

\section{References}

Andrew R. L., Peakall R., Wallis I. R., WoOd J. T., Knight E. J., and FOLEY W. J. 2005. Marker-based quantitative genetics in the wild: The heritability and genetic correlation of chemical defenses in Eucalyptus. Genetics 171:1989-1998.

Andrew R. L., Peakall R., Wallis I. R., and Foley W. J. 2007. Spatial distribution of defense chemicals and markers and the maintenance of chemical variation. Ecology 88:716-728.

BECERRA J. X. 2007. The impact of herbivore-plant coevolution on plant community structure. Proc. Natl. Acad. Sci. USA 104:7483-7488.

BraithWAite L. W., DUDZINSKI M. L., and TURNER J. 1983. Studies on the arboreal marsupial fauna of eucalypt forests being harvested for woodpulp at Eden, NSW. 2. Relationship between the fauna density, richness and diversity, and measured variables of habitat. Aust. Wildl. Res. 10:231-247.

ChilcotT M. J., and HumE I. D. 1984. Nitrogen and urea metabolism and nitrogen requirements of the common ringtail possum, Pseudocheirus peregrinus, fed Eucalyptus andrewsii foliage. Aust. J. Zool. 32:615-622.

Choo G. M., Waterman P. G., McKey D. B., and Gartlan J. S. 1981. A simple enzyme assay for dry matter digestibility and its value in studying food selection by generalist herbivores. Oecologia 49:170-178. 
CORK S. J., and FOLEY W. J. 1991. Digestive and metabolic strategies of arboreal mammalian folivores in relation to chemical defenses in temperate and tropical forests, pp. 133-166, in R. T. Palo and C. T. Robbins (eds.). Plant Chemical Defenses and Mammalian Herbivory. CRC Press, Boca Raton.

Cunningham R. B., Pope M. L., and LindenMaYer D. B. 2004. Patch use by the greater glider (Petauroides volans) in a fragmented forest ecosystem. III. Night-time use of trees. Wildl. Res. 31:579-585.

DeGabriel J. L., Wallis I. R., Moore B. D., and Foley W. J. 2008. A simple, integrative assay to quantify nutritional quality of browses for herbivores. Oecologia 156:107-116.

Degabriel J. L., Moore B. D., Foley W. J., and Johnson C. N. 2009. The effects of plant defensive chemistry on nutrient availability predict reproductive success in a mammal. Ecology 90:711-719.

Dyckmans J., Flessa H., Brinkmann K., Mai C., and Polle A. 2002. Carbon and nitrogen dynamics in acid detergent fibre lignins of beech (Fagus sylvatica L.) during the growth phase. Plant Cell Environ. 25:469-478.

EHRLICH P. R., and RAVEN P. H. 1964. Butterflies and plants: A study in coevolution. Evolution 18:586-608.

ElisseEFF A., and PONTIL M. 2002. Leave-one-out error and stability of learning algorithms with applications. pp. 415 in J.A.K. Suykens (ed.). Advances in Learning Theory: Methods, Models, and Applications. IOS Press, Leuven.

Eschler B. M., PASS D. M., WAllis I. R., and Foley W. J. 2000. Distribution of foliar formylated phloroglucinol derivatives amongst Eucalyptus species. Biochem. Syst. Ecol. 28:813-824.

FoleY W. J., and HUME I. D. 1987. Nitrogen requirements and urea metabolism in 2 arboreal marsupials, the greater glider (Petauroides volans) and the brushtail possum (Trichosurus vulpecula), fed eucalyptus foliage. Physiol. Zool. 60:241-250.

FutUYMA D. J. 2000. Some current approaches to the evolution of plant-herbivore interactions. Plant Species Biol. 15:1-9.

GleadOW R. M., FolEy W. J., and WoOdROW I. E. 1998. Enhanced $\mathrm{CO}_{2}$ alters the relationship between photosynthesis and defense in cyanogenic Eucalyptus cladocalyx. Plant Cell Environ. 21:12-22.

Hollander M., and WOLFE D. 1999. Nonparametric Statistical Methods. Wiley, New York.

JACKSON S.M. 2000. Glide angle in the genus Petaurus and a review of gliding in mammals. Mamm. Rev. 30:9-30.

Kavanagh R. P., and Lambert M. J. 1990. Food selection by the greater glider, Petauroides volans - is foliar nitrogen a determinant of habitat quality? Aust. Wildl. Res. 17:285-299.

Knepp R. G., Hamilton J. G., Mohan J. E., Zangerl A. R., Berenbaum M. R., and DeLuCia E. H. 2005. Elevated $\mathrm{CO}_{2}$ reduces leaf damage by insect herbivores in a forest community. New Phytol. 167:207-218.

LAWLer I. R., Foley W. J., Woodrow I. E., and CorK S. J. 1997. The effects of elevated $\mathrm{CO}_{2}$ atmospheres on the nutritional quality of Eucalyptus foliage and its interaction with soil nutrient and light availability. Oecologia 109:59-68.

Lawler I. R., Foley W. J., Eschler B. M., Pass D. M., and HANDASYDE K. 1998a. Intraspecific variation in Eucalyptus secondary metabolites determines food intake by folivorous marsupials. Oecologia 116:160-169.

LAWler I. R., Foley W., PASS D. M., and Eschler B. M. 1998b. Administration of a $5-\mathrm{HT}_{3}$ receptor antagonist increases the intake of diets containing Eucalyptus secondary metabolites by marsupials. J. Comp. Physiol. B 168:611-618.

LINDENMAYER D. B. 1997. Differences in the biology and ecology of arboreal marsupials in forests of southeastern Australia. $J$. Mammal. 78:1117-1127.

Lindenmayer D. B., Cunningham R. B., and Pope M. L. 1999. A large-scale "experiment" to examine the effects of landscape context and habitat fragmentation on mammals. Biol. Conserv. 88:387-403.

LINDROTH, R. L. 2010. Impacts of elevated atmospheric $\mathrm{CO}_{2}$ and $\mathrm{O}_{3}$ on forests: Phytochemistry, trophic interactions, and ecosystem dynamics. J. Chem. Ecol. 36:2-21.

Mansfield J. L., Curtis P. S., ZAK D. R., and Pregitzer K. S. 1999. Genotypic variation for condensed tannin production in trembling aspen (Populus tremuloides, Salicaceae) under elevated $\mathrm{CO}_{2}$ and in high- and low-fertility soil. Am. J. Bot. 86:1154-1159.

Marsh K. J., Foley W. J., Cowling A., and Wallis I. R. 2003a. Differential susceptibility to Eucalyptus secondary compounds explains feeding by the common ringtail (Pseudocheirus peregrinus) and common brushtail possum (Trichosurus vulpecula). J. Comp. Physiol. B 173:69-78.

MARSH K. J., WALLIS I .R., and FOLEY W. J. 2003b. The effect of inactivating tannins on the intake of Eucalyptus foliage by a specialist Eucalyptus folivore (Pseudocheirus peregrinus) and a generalist herbivore (Trichosurus vulpecula). Aust. J. Zool. 51:31-42.

Marsh K. J., Wallis I. R., ANDrew R., and Foley W. J. 2006. The detoxification limitation hypothesis: Where did it come from and where is it going? J. Chem. Ecol. 32:1247-1266.

MCARTHUR C., and SANSON G. D. 1991. Effects of tannins on digestion in the common ringtail possum (Pseudocheirus peregrinus), a specialized marsupial folivore. J. Zool. 225:233-251.

McIlwee A. M., LAWler I. R., CorK S. J., and Foley W. J. 2001. Coping with chemical complexity in mammal-plant interactions: near-infrared spectroscopy as a predictor of Eucalyptus foliar nutrients and of the feeding rates of folivorous marsupials. Oecologia 128:539-548.

Moore B. D., and Foley W. J. 2005. Tree use by koalas in a chemically complex landscape. Nature 435:488-490.

Moore B. D., Wallis I. R., MARSh K. J., and FoleY W. J. 2004. The role of nutrition in the conservation of the marsupial folivores of eucalypt forests, pp. 549-575, in D. Lunny (ed.). Conservation of Australia's Forest Fauna. CSIRO, Collingwood.

Moore B. D., Foley W. J., Wallis I. R., Cowling A., and HANDASYDE K. A. 2005. Eucalyptus foliar chemistry explains selective feeding by koalas. Biol. Lett. 1:64-67.

Moore B. D., LAWler I. R., Wallis I. R., Beale C. M., and Foley W. J. 2010. Palatability mapping: A koala's eye view of spatial variation in habitat quality. Ecology 91:3165-3176.

OSAWA R., and SLY L. I. 1992. Occurrence of tannin-protein complex degrading Streptococcus sp. in feces of various animals. Syst. Appl. Microbiol. 15:144-147.

Osborne B. G., Fearn T., Hindle P. T., and Osborne B. G. 1993. Practical NIR Spectroscopy with Applications in Food and Beverage Analysis. Longman Publishing Group, Essex.

Pinto, D. M., Blande, J. D., Souza, S. R., NerG, A.-M., and Holopainen, J. K. 2010. Plant volatile organic compounds (VOCs) in ozone $\left(\mathrm{O}_{3}\right)$ polluted atmospheres: The ecological effects. J. Chem. Ecol. 36:22-34.

Pope M. L., Lindenmayer D.B., and Cunningham R. B. 2004. Patch use by the greater glider (Petauroides volans) in a fragmented forest ecosystem. I. Home range size and movements. Wildl. Res. 31:559-568.

PRYOR L. D. 1959. Species distribution and association in Eucalyptus. pp. 461-471, in Keast A., Crocker R. L., and Christian C. S. (eds.). Biogeography and Ecology in Australia. Junk, The Hague.

RAUSHER M. D. 2001. Coevolution and plant resistance to natural enemies. Nature 411:857-864.

SCRIVENER N. J., JOHNSON C. N., WALLIS I. R., TAKASAKI M., FOLEY W. J., and KrockenBerger A. K. 2004. Which trees do wild common brushtail possums (Trichosurus vulpecula) prefer? Problems and solutions in scaling laboratory findings to diet selection in the field. Evol. Ecol. Res. 6:77-87. 
ShenK J. S., and Westerhaus M. O. 1991. Population structuring of near-infrared spectra and modified partial least-squares regression. Crop Sci. 31:1548-1555.

Shipley L. A., Forbey J. S., and Moore B. D. 2009. Revisiting the dietary niche: when is a mammalian herbivore a specialist? Integr. Comp. Biol. 49:274-290.

Silanikove N., Gilboa N., Nir I., Pervolotsky A., and Nitsan Z. 1996. Effect of a daily supplementation of polyethylene glycol on intake and digestion of tannin-containing leaves (Quercus calliprinos, Pistacia lentiscus, and Ceratonia siliqua) by goats. J. Agr. Food Chem. 44:199-205.

StePhens D. W., and KrebS J.R. 1986. Foraging Theory. Princeton University Press, Princeton.

THOMPSON J. N. 1994. The Coevolutionary Process. University of Chicago Press, Chicago.
WALlis I. R., and Foley W. J. 2005. The rapid determination of sideroxylonals in Eucalyptus foliage by extraction with sonication followed by HPLC. Phytochem. Anal. 16:49-54.

Wallis I. R., Watson M. L., and Foley W. J. 2002. Secondary metabolites in Eucalyptus melliodora: field distribution and laboratory feeding choices by a generalist herbivore, the common brushtail possum. Aust. J. Zool. 50:507-519.

Wallis I. R., Nicolle D., and Foley W. J. 2010. Available and not total nitrogen in leaves explains key chemical differences between the eucalypt subgenera. For. Ecol. Manag. 260:814821

Zvereva E. L., and Kozlov M. V. 2006. Consequences of simultaneous elevation of carbon dioxide and temperature for plant-herbivore interactions: a meta-analysis. Glob. Change Biol. $12: 27-41$. 